\title{
La sombra conceptual. Conceptos operativos e indicadores formales en la fenomenología
}

\section{The Conceptual Shadow. Operational Concepts and Formal Indicators in Phenomenology}

\author{
ÁNGEL XOLOCOTZI YÁÑEZ \\ BENEMÉRITA UNIVERSIDAD AUTÓNOMA DE PUEBLA
}

Recibido: 20/05/2019 Aceptado:05/06/2019

\section{RESUMEN}

En el siguiente escrito buscamos poner de manifiesto las necesidades expresivas, en razón de las cuales, el joven Heidegger emprende el camino en búsqueda de los indicadores formales. Nuestra línea argumentativa toma como punto de partida la crítica que Eugen Fink construye alrededor de los conceptos de la fenomenología husserliana, pero se enfoca exclusivamente en la transformación hermenéutica de sus contenidos. Con ello pretendemos aclarar la transición de qué al cómo que caracteriza la propuesta fenomenológico-hermenéutica de Martin Heidegger, desde el punto de vista de su conceptualidad específica.

\author{
PALABRAS CLAVE
}

LENGUAJE, SIGNIFICATIVIDAD, FACTICIDAD, CONCEPTOS, OCULTACIÓN

\begin{abstract}
In the following text we aim to expose the expressive needs, in view of which the young Heidegger opens his investigation in direction of the formal indications. Our line of argument takes as its starting point the critics that Eugen Fink directs against the concepts of husserlian phenomenology, but focuses exclusively on the hermeneutic transformation of its contents.
\end{abstract}

(C) Contrastes. Revista Internacional de Filosofía, vol. XXIV-Nº3 (2019), pp. 73-90. ISSN: 1136-4076 Departamento de Filosofía, Universidad de Málaga, Facultad de Filosofía y Letras Campus de Teatinos, E-29071 Málaga (España) 
Thereby we intent to clarify the transition from the what to how -that characterizes the hermeneutic-phenomenological proposal, from the point of view of its specific conceptuality.

KEYWORDS

LANGUAGE, FACTICITY, CONCEPTS, CONCEALMENT

\section{INTRODUCCIÓN}

TODA REFLEXIÓN FILOSÓFICA TIENE la posibilidad de relacionarse consigo misma a nivel expresivo; sin embargo, no toda filosofía admite como tarea la revisión de los conceptos con los que trabaja. Esta decisión depende de la noción de lenguaje que se tome como punto de partida. Si de entrada se considera la forma gramatical como una neutralidad invisible e improductiva que se limita a reflejar los pensamientos en un registro lingüístico, y que por lo tanto, tiene un papel pasivo en los procesos de reflexión, entonces la filosofía entendida como un decir verdadero se mantendría independiente de las modalidades fácticas de este decir. Por el contrario, las filosofías que parten del hecho de que sus «objetos» tienen lugar en el elemento del concepto, reivindican la necesidad de regresar a las operaciones lingüísticas mediante las cuales se expresan; motivadas, en gran medida, por la sospecha de que no existe un lenguaje neutral y transparente que sirva de herramienta para exteriorizar pensamientos y estados de ánimo; sino que todo lenguaje, en tanto que medio único del pensamiento, también interviene en los procesos de formación de sentido ( $c f$. Gadamer, 2004).

Esto último no quiere decir que un problema filosófico pueda resolverse mediante la puesta al descubierto de sus medios y condiciones materiales. La necesidad de preguntarse por el momento expresivo de una filosofía surge, mas bien, de la relación que ésta mantiene con sus propios límites. En el caso concreto de la fenomenología, la tarea de una revisión de los conceptos con los que se expresa, forma parte de la exigencia metodológica de trabajar sin presupuestos metafísicos, físicos, psicológicos y lingüísticos; pero no constituye una tarea previa, puesto que dichos conceptos deben surgir de la pregunta correspondiente, de modo que sea el problema y no el lenguaje el hilo conductor de sus desarrollos.

Así, lejos de reducir su campo de estudio al sentido lingüístico, el proyecto fenomenológico se define en primer lugar por la voluntad de expresar, de verbalizar, el sentido mismo de la experiencia silenciosa, intuitiva y silenciosa de la conciencia intencional. [...] Si tiene sentido hablar de un lenguaje fenomenológico que se oponga al lenguaje natural y ordinario, es porque la fenomenología se encarga de expresar el «sentido» silencioso, silencioso y por tanto no lingüístico de la experiencia concreta [...]. Y el reto es encontrar metáforas, analogías que nos permitan expresar, de manera lingüística, ese 
significado que, precisamente, está por encima y por debajo de cualquier lenguaje natural y ordinario (Grondin, 2010, pp. 256-257).

Esto quiere decir que cuando los conceptos de la fenomenología son puestos en tela de juicio, no se investiga únicamente cómo es que fueron formados o de dónde provienen; también y sobre todo, se investiga aquello que se configura a través de ellos. En el caso de la propuesta heideggeriana -motivo central del presente trabajo, la pregunta acerca de sus medios lingüísticos irrumpe en el plano de la confrontación con la fenomenología de Husserl y se inscribe específicamente en el proceso de crítica y transformación de sus contenidos. Dado que éstos no son objetos o estados de cosas ya constituidos, sino que remiten a la vivencia del mundo circundante que nosotros mismos experimentamos de manera absorta en la ocupación cotidiana; la conceptualidad específica para abordarlos no puede estar orientada hacia el paradigma de una investigación pura y formal. En respuesta a las necesidades expresivas de sus propios hallazgos fenomenológicos, Heidegger hablará entre 1920 y 1923 de los indicadores formales (formale Anzeige) es decir, de conceptos que no precisan del cumplimiento intuitivo puesto que de entrada no comunican contenidos; sino que se llevan a cabo como una especie de praxis fenomenológica, esto es, como un comportamiento de frente a lo dado.

En el presente escrito buscamos poner de manifiesto el proceso, mediante el cual, el joven Heidegger llega a tal decisión conceptual. Nuestra línea argumentativa toma como punto de partida la crítica que Eugen Fink dirige en contra de los conceptos de la fenomenología husserliana, pero se enfoca exclusivamente en la transformación hermenéutica de sus contenidos. Con ello pretendemos aclarar la transición de qué al cómo que caracteriza la propuesta heideggeriana desde el punto de vista de su conceptualidad específica. El itinerario que seguiremos comprende, en primer lugar, la distinción entre los conceptos temáticos y operatorios de la fenomenología; para dar paso, en segundo lugar, a la integración de los escorzos o sombras operatorias de un nivel lingüístico a un nivel de contenido. Por último, buscamos reconectar dichos contenidos operatorios con su nivel expresivo mediante la búsqueda de los indicadores formales, es decir, de conceptos filosóficos capaces de traer al lenguaje aquello que tiende a ocultarse.

\section{CONCEPTOS TEMÁTICOS Y OPERATORIOS EN LA FENOMENOLOGÍA}

Al igual que toda indagación teorética, la fenomenología parte del factum de los lenguajes para expresar sus hallazgos (Husserl, 1999a, p. 217 y p. 230); sin embargo, a diferencia del científico, el fenomenólogo deberá fundamentar sus conceptos o unidades de pensamiento en una fuente legítima pre-lingüística del conocimiento que garantice su comprensibilidad. En este 
ámbito no basta con fijar y determinar ciertos términos de tal modo que estos cumplan con funciones bien definidas del conocimiento; también hay que retrotraerlos a estructuras lógicas puras, que, como tales, pueden ser intuidas en la abstracción ideativa (Husserl, 1999b, p. 464 y ss.). Durante la formulación de conceptos en el ámbito fenomenológico se avanza, así, en dirección contraria a la comprensión natural simbólica de las palabras y se dirige al ámbito ante-predicativo de intuición, buscando que toda diferencia terminológica corresponda realmente a las diferencias entre las cosas ahí dadas. En este sentido, Husserl señala lo siguiente:

Vemos con evidencia, en efecto, que ninguna teoría podría sacar su propia verdad si no es de los datos originarios [dados en la intuición]. Toda proposición que no hace mas que dar expresión a semejantes datos, limitándose a explicitarlos por medio de significaciones fielmente ajustadas a ellos, es también realmente, como hemos dicho en las palabras iniciales de este capítulo, un comienzo absoluto, llamado a servir de fundamento en el genuino sentido del término, es realmente un principium (Husserl, 1962, p. 58).

Como podemos observar, para Husserl «la vestidura gramática» que sirve de soporte a la fenomenología en tanto que disciplina descriptiva posee cierto carácter transparente, pues aún cuando «las palabras utilizadas pueden proceder del lenguaje vulgar y ser equívocas o vagas por obra de su cambiante sentido», sirven para fijar y comunicar lo ganado en la actitud trascendental. «En la medida en que se 'corresponden', en la forma de la expresión actual, con lo intuitivamente dado, cobran un sentido determinado, que es su claro sentido actual hic et nunc, y partiendo de aquí pueden fijarse científicamente» (Husserl, 1962, p. 150). Esto quiere decir que al expresar sus resultados, la actitud trascendental también trastocaría el lenguaje mundano; y que la palabra, convertida ya en concepto fenomenológico, estaría libre de significaciones obscuras y ambiguas.

El primero en señalar los problemas que se derivan de esta postura fue Eugen Fink, alumno y asistente de Husserl en sus últimos años de vida. Para Fink, el empleo de la palabra como «fiel adaptación a la esencia intuida» garantiza el ingreso del lenguaje al ámbito fenomenológico, pero únicamente en tanto que portador de sentido, negando al mismo tiempo su carácter constituyente. Sin embargo, dado que la fenomenología y la filosofía en general parten de una comprensión conceptual previa y a partir de ella determinan su propio vocabulario, es decir, en vistas de que sus conceptos han sido fijados de manera aproximativa mediante otros conceptos imprecisos; una considerable cantidad de términos fundamentales permanecen impregnados de sombras y escorzos de sentido desde el momento de su definición. 
Para tematizar este exceso de sentido que se filtra a través del lenguaje, Fink introducirá, en su célebre conferencia titulada Conceptos operatorios en la fenomenología husserliana (1968), la distinción entre la función operatoria y la función temática de los conceptos filosóficos: mientras que los conceptos operatorios son tomados del lenguaje natural y empleados de manera inadvertida, mediante los conceptos temáticos, «el pensamiento fija y custodia lo pensado». Como ejemplos del segundo grupo menciona los conceptos de Idea, Ousía, Mónada, Espíritu, Voluntad de Poder y Subjetividad trascendental. Al primer grupo, en cambio, pertenecen metáforas, analogías, modelos de pensamiento, esquemas intelectuales y otras formulaciones no reflexionadas, que pese a sus efectos imprevisibles e incontrolables sobre el contenido filosófico, son inevitables.

Por un lado, esto quiere decir que el oscurecimiento que impregna el despliegue del pensamiento filosófico no es producto de intenciones de significado fallidas, sino que pertenecen a la naturaleza del medio lingüístico. Por otro lado, esto implica que la anhelada claridad y distinción filosóficas son posibles pero sólo en cierto grado, debido a que el uso de conceptos es siempre impreciso y da lugar a sombras de sentido inadvertidas. Los doce significados de Vorstellung al final de la quinta investigación lógica (Vongehr, 2005, p.7), el concepto de constitución, que oscila entre Sinnbildung y Kreation, o bien, términos como epojé y subjetividad trascendental, que son utilizados de «manera operativa» más de lo que han sido aclarados «temáticamente», son ejemplos de que incluso en el más alto grado de reflexividad, el pensamiento «está impregnado de sombras» (Fink, 1968, p. 204).

Pero, ¿sería posible reemplazar el uso ingenuo y natural de la palabra por otro completamente trascendental? En principio, la reducción fenomenológica bastaría para limitar y eliminar la tensión entre los conceptos temáticos y operativos, sin embargo, para su expresión, el fenomenólogo cuenta únicamente con el lenguaje convencional, cuyo uso precisaría nuevamente de una aclaración temática porque aún no ha sido depurado de intuiciones remotas, confusas e impropias (Fink, 1968, p. 198).

Debido a la cadena infinita que se desata a nivel lingüístico, el fenomenólogo que tomara como punto de partida la tarea de crear un lenguaje más preciso, jamás lograría pasar de las «meras palabras» a «las cosas mismas». Aunque a decir verdad, en el ámbito fenomenológico no se pretende llegar a la definición universal, transparente e irrevocable de sus términos y conceptos, tal como sucede con la geometría (Husserl 1962, p.165). Por el contrario, las descripciones, los términos y los conceptos con los que opera la fenomenología deben permanecer «en estado de fluidez, o continuamente a punto de diferenciarse con arreglo a los progresos del análisis de la conciencia y del descubrimiento de nuevas capas fenomenológicas dentro de lo intuido 
primeramente en una unidad indiferenciada» (Husserl, 1962, p. 201). Por otro lado, en vistas de que esta disciplina describe lo dado, tal y como ha sido dado, es decir, sin prejuicios y sin creencias previas, la comprensión de sus conceptos estaría igualmente garantizada por medio de la designación intuitiva (Vongehr, 2005, p. 5), de modo que si se llegasen a dar dificultades o confusiones, estas se hallarían «en la dirección antinatural de la intuición y del pensamiento, que exige el análisis fenomenológico» (Husserl, 1999a, p. 221).

Por estas razones, Husserl tomará por «exageradas» las observaciones que Fink le hizo acerca de las posibilidades expresivas de la fenomenología; no obstante, el interés de Fink por la naturaleza de lenguaje filosófico no es analítico, pragmático ni enteramente fenomenológico. Esto quiere decir que sus señalamientos no se insertan en el mismo nivel de análisis que su maestro, sino que tiene lugar en el marco de una doctrina trascendental del método, cuyo desarrollo conduciría a «deshacer las ingenuidades implícitas en la primera fase de la fenomenología, de entre ellas 'la aporía básica de la utilización de un lenguaje referido a las cosas y a los entes, para determinar el ámbito en el que surgen las cosas y los entes'» (San Martín, 1990, p. 250). De este modo, mientras que Husserl emprende el descenso hacia la experiencia antepredicativa tomando como base la percepción pura ( $c f$. Husserl, 1980, parágrafos 13 y 15); Fink, "grandemente influenciado por la tematización heideggeriana de la facticidad» apunta al campo previo a toda estrategia objetivizante que hace posible la distinción entre actitud natural y actitud trascendental ( $c f$. Schütz, 1971).

En última instancia, mediante la crítica a los conceptos fundamentales de la fenomenología se hace patente la confrontación entre dos maneras de concebir el quehacer de esta disciplina. Por un lado, tal parece que la fenomenología entendida como una ciencia descriptiva que indaga en el campo de la conciencia pura trascendental y de la intuición pura no está obligada a esclarecer la posibilidad de sus medios lingüísticos, debido a que el cumplimiento intuitivo de la intención significativa que anima sus conceptos, garantizaría la ausencia de ambigüedades y equívocos a nivel conceptual. Pero por otro lado, también es cierto que todo pensamiento filosófico está confinado a su formulación y transmisión verbal, y que todo filosofema puede ser tomado, en última instancia, como documento literario; lo cual vuelve ineludible la tarea de repensar la relación que la filosofía guarda con sus propios conceptos.

Sin embargo, cabe recalcar que Fink no busca liberar a la fenomenología de las sombras del pensamiento mediante la puesta al descubierto de sus conceptos operativos, en primer lugar, porque todo concepto visto bajo la luz del análisis ya no aparece en su función operatoria, sino que se convierte en un tema, cuyo tratamiento precisa nuevamente de conceptos opacos e imprecisos. 
En segundo lugar, porque aspirar a la total claridad lingüística parece una empresa imposible, más no por una deficiencia del lenguaje o de capacidad cognoscitiva humana, sino porque la filosofía en tanto comportamiento fundamental del ser humano no puede llevarse a cabo como una «transparente posesión de la verdad». Por el contrario:

[...] la manera humana de concebir el ente en su conjunto se produce en una captación de la totalidad, pero jamás de tal manera que el todo se abriría a un concepto universal plenamente aclarado y sin sombras. La captación humana del mundo piensa la totalidad en un concepto temático del mundo, que sin embargo es una perspectiva finita, pues, en su formulación, son utilizados conceptos que se mantienen en la sombra (Fink, 1968, p. 195).

Las sombras que acompañan toda conceptualización no son, pues, lapsus o errores que el pensador, con ayuda de la hermenéutica o de una tematización más amplia, pudiera aclarar más tarde; sino que forman parte esencial de la actividad filosófica finita, de modo que «cuanto más profunda es la fuerza que intenta operar un esclarecimiento, más profunda también es la sombra que acompaña a los conceptos fundamentales»(Fink, 1968, p. 204). Y aún más, para Fink: «la fuerza iluminadora del pensamiento se nutre de lo que permanece en la sombra [...]. Tiene su élan productivo en el empleo irreflexivo de esos conceptos cubiertos de sombra» (Fink, 1968, p. 195).

\section{LAS SOMBRAS CONCEPTUALES.}

La crítica a la conceptualiadad fenomenológica no reflexionada que en un principio estaba dirigida a Husserl, también se comprueba en Heidegger, sobre todo y de manera ejemplar en «la explicación temática del ser, bajo la claridad comprensora del tiempo e inversamente» (Fink, 1968, p. 197). Sin embargo, en el caso de la propuesta heideggeriana ocurre un giro interesante, pues en lugar de aspirar a la total claridad, las sombras serán asumidas como elementos constitutivos del contenido filosófico. El máximo grado de esta admisión se encuentra en la interpretación de la verdad, a la cual le pertenece una contraverdad interior, una no-verdad que da inicio al juego entre la ocultación y la desocultación. No obstante, antes de que la pregunta por el ser devenga en la pregunta por la verdad del ser, y antes de que el ser mismo sea definido en términos de sustracción y olvido; encontramos indicios de otra forma de integración que conciernen directamente a los «objetos» de la fenomenología de los que habla Husserl. Nos referimos aquí a la tematización del fenómeno 
de la significatividad mundana como resultado de la apropiación hermenéutica del lema de la fenomenología «a las cosas mismas» ( $c f$. Heidegger, 2006b, p. 103 y ss.). ${ }^{1}$

Localizar el origen de las sombras operatorias no ya en el plano del concepto, sino del contenido fenomenológico, es posible sobre la base de una interpretación radical de los «objetos» de la fenomenología. ${ }^{2}$ Mientras que para Husserl «las cosas mismas» son accesibles únicamente mediante la puesta entre paréntesis de la actitud natural, en la cual experimentamos el mundo como «terminado», sin poder dar cuenta de los procesos de constitución; la fenomenología hermenéutica desarrollada por Heidegger se enfoca en lo que, por así decirlo, ha sido puesto entre paréntesis. De hecho, en la lección del semestre de invierno de 1923/24 titulada Introducción a la investigación fenomenológica, señala que «lo característico» del proyecto husserliano «se encuentra en que lo que debe ser conocido en este conocimiento, es, desde el principio, secundario» (2006a, p. 108).

Con «lo que debe ser conocido» Heidegger no se refiere a las cosas de la fenomenología desde el punto de vista de su constitución y validez, sino a las cosas que vienen a nuestro encuentro como problemas prefigurados ya en determinadas direcciones por la existencia fáctica:

Esto se funda en el hecho de que 'a las cosas mismas' ya no pueda querer decir aquí: traer ante la mente las cosas libremente, por sí mismas, antes de un específico modo de preguntar, sino que quiere decir: dejar que vengan a nuestro encuentro, dentro de la problemática prefigurada de modo totalmente especifico (Heidegger, 2006a, p. 109).

Así, en un intento por definir su propuesta frente a la fenomenología orientada hacia el campo de la conciencia pura, Heidegger hará de «lo conocido» el objeto central de su investigación. Sin embargo, con el énfasis en el contenido, esto es, en el qué de la fenomenología, no apunta a un núcleo, a una esencia o a un quid que deba ser desmantelado con ayuda de la Destruktion. La identificación del qué con contenidos fijados y encubiertos por la tradición metafísica no aparecerá en el pensamiento heideggeriano sino hasta la lección del semestre de verano de 1925 titulada Prolegómenos para

$1 C f$. al respecto la discusión que Xolocotzi y Zirión (2018) sostienen sobre el lema de la fenomenología.

2 Según la interpretación que Heidegger lleva a cabo en Ontología. Hermenéutica de la facticidad, las Investigaciones lógicas de Husserl «son investigaciones sobre objetos tradicionalmente asignados al campo de la lógica. [...] Si lo que la lógica dice ha de tener fundamento alguno, es necesario que esas cosas de las que habla sean en sí mismas accesibles» (1999b, p. 94). 
una historia del concepto de tiempo (2006b). Cuando Heidegger habla aquí de la necesidad de reivindicar la primacía del qué sobre el cómo fenomenológicos, se está dirigiendo en contra de una idea determinada de fenomenología que, emulando el ideal metódico de la matemática, deja aparecer las cosas desde una determinada dirección del cómo, esto es, desde el punto de vista de la teoría, lo cual conduciría a la pérdida de las cosas en su libre donación (Heidegger, 2006b, p. 126 y ss.) ${ }^{3}$

Ya a partir de 1925 y bajo la luz de una renovada crítica de la propuesta de su maestro Husserl -esta vez centrada en el momento de la reducción fenomenológica-, la interpretación heideggeriana de los contenidos fenomenológicos experimentará un vuelco. Esta vez, el qué de la fenomenología será identificado con una idea determinada de ser-objeto, que presupone la ruptura con su contexto mundano y que precisa de las estructuras estratificadas de los actos de la conciencia para su explicación. Por el contrario, el cómo será identificado en tanto el «modo primario» del aparecer de «las cosas», que se muestra de manera pre-temática en la ocupación con el mundo circundante, y que precisamente por ello, es accesible en su más fáctico para-qué (cf. Baur, 2010).

En el parágrafo 15 de Ser y tiempo, Heidegger profundiza aún más en esta idea cuando aclara que si el tema de su investigación es el ser, el «objeto» que antecede fenomenológicamente a dicho tema, es decir, «el terreno fenoménico preliminar», no puede considerarse propiamente objeto porque no es accesible a partir del conocimiento teórico (Heidegger, 2003, p. 89). ¿Cuál es, entonces, el ente que constituye el «tema previo» de la fenomenología? Se responderá: las cosas en el sentido griego de $\pi \rho \alpha ́ \gamma \mu \alpha \tau \alpha$, esto es: «aquello con lo que uno tiene que habérselas en el trato de la ocupación (en la $\left.\pi \rho \tilde{\alpha} \xi_{1 \zeta}\right) »$ (2003, p. 90). Dado que «las cosas» en tanto útiles se muestran como tales únicamente mientras están siendo utilizadas para tal o cual fin, su sentido llega a cumplimiento en su específico para-qué: para escribir, para trabajar, para viajar, para medir, etc. ${ }^{4}$

Ahora bien, que el martillo se muestre genuinamente en el martillar, esto es, que el ente comparezca «desde él mismo en la ocupación y para ella», quiere decir que su modo de ser es accesible precisamente cuando no está siendo tematizado en cuanto útil. De hecho, «lo peculiar de lo inmediatamente

3 En todo caso, Heidegger no deja de reconocer que la fenomenología en cuanto método es el cómo de la investigación filosófica ( $c f$. Heidegger, 1999, p. 97).

4 La orientación hermenéutica de "las cosas" de la fenomenología en tanto un dinámico ser-para, también afectará al cómo del aparecer y al sujeto de la experiencia. Por ello, el desarrollo reflexivo de la esfera trascendental de las vivencias deberá transformarse en comprensión hermenéutica, la cual, a su vez, exige como punto de partida la forma concreta y finita del sujeto en su existencia fáctica (Dasein). 
a la mano consiste en retirarse, por así decirlo, 'a' su estar a la mano para estar con propiedad a la mano» (Heidegger, 2003, p. 91). Ni siquiera en la circunspección (Umsicht), que es el modo de ver del comportamiento práctico, dicho ente comparece de modo temático. Por el contrario, cuando el útil «llama la atención» debido, por ejemplo, a su descompostura o a su ausencia, ya no aparece en su estar a la mano, sino que se revela como un mero estarahí en su inutilidad, que sin ser todavía un objeto de contemplación teórica, pone de relieve al mundo circundante en su para-qué y en su con-qué faltantes (Heidegger, 2003, pp. 92-97).

Pero la comprensión operatoria o precomprensión que sirve de acceso fenomenológico no es exclusiva del ente intramundano, sino que se extiende al mundo circundante, es decir, al "mundo mas cercano al Dasein cotidiano" (Heidegger, 2003, p. 88). Esto se hace patente cuando, en punto decisivo de la argumentación, Heidegger afirma que «el no-acusarse [sich-nicht-melden] del mundo es la condición de posibilidad para que lo a la mano salga en su nollamatividad. Y ello constituye la estructura fenoménica del ser-en-sí de este ente» (2003, p. 97). ${ }^{6}$ Por un lado, esta afirmación implica que la apertura del mundo en su carácter significativo es posible sobre la base del ocultamiento operativo de los entes intramundanos. Por otro lado, que el mundo en su carácter de abierto dependa de la ocupación circunspecta con el útil, también significa que el mundo deviene tal desde la sombra de lo no tematizado. ${ }^{7}$

Si el no llamar la atención, el contenerse, el permanecer bajo la sombra de la operatividad constituye el modo de aparecer tanto del ser a la mano, como del fenómeno del mundo circundante, entonces ¿cómo es que se puede hablar sobre ellos sin convertirlos en tema?, ¿cómo abordarlos

5 La comprensión de mundo circundante, esto es, del mundo que se muestra desde el estar ocupado, es preontológica porque se lleva a cabo conforme al mundo (Weltmässig). Por el contrario, en un nivel ontológico, el fenómeno del mundo se muestra en cuanto mundo, es decir, en su mundaneidad (Weltlichkeit). En la presente exposición hemos intentado mantenernos en el nivel pre-fenomenológico de la mundicidad del mundo, pero es inevitable que nuestros desarrollos conduzcan finalmente a la mundaneidad el mundoel camino de y hacia... ana el espacio para su ejecucion to que le otorga al lenguaje un papel designativo, para ecto positivo.

6 «El peculiar y obvio «en-sí» de las cosas comparece en la ocupación que hace uso de estas «cosas» sin advertirlas expresamente...» (Heidegger, 2003, p. 96).

7 De acuerdo con los fines del presente artículo nos hemos concentrado en la estructura operativa que caracteriza la comparecencia del útil y del mundo; sin embargo, para Heidegger, la experiencia fenomenológica en general se mueve en términos de manifestación y ocultamiento, debido a que tiene lugar en el cruce entre «lo ya dado» ónticamente y aquello que hace posible esta dación, esto es, el horizonte de «lo pre-dado» ontológicamente. Al respecto véase Basso (2019), Rodríguez (2015) y Xolocotzi (2004). 
fenomenológicamente sin irrumpir en esta fluctuación del claroscuro propio de su aparecer? La integración de la operatividad como elemento constitutivo del mostrarse fenomenológico exigirá una nueva conceptualidad, que en lugar de apelar a estructuras fijas propias de la orientación teorética, busque esclarecer la dinámica mundana en su ejecución performativa. En lo que resta nos enfocaremos en el camino que Heidegger emprende en busca de «conceptos no objetivantes, sino situacionales», que sean capaces de traer al lenguaje la experiencia de lo que no se da sobre la base de intuiciones plenamente desenvueltas; sino que, por el contrario, aparece de manera auténtica únicamente desde su ocultamiento operativo.

\section{EL PAPEL DE LOS INDICADORES FORMALES}

La preocupación de Heidegger por el lenguaje con el que se expresaría su propia filosofía alcanzará su punto culmen con la proclamación de la tarea de liberar al lenguaje de la lógica y la gramática occidentales, pues las considera formas en las que la «metafísica» se adueñó de él desde hace tiempo (2000a, p. 260). De hecho, en la Carta sobre el humanismo indica que la tercera sección de la primera parte de Ser y tiempo no se dio a la imprenta precisamente porque «el pensar no fue capaz de expresar ese giro con un decir de suficiente alcance ni tampoco consiguió superar esa dificultad con ayuda del lenguaje de la metafísica» (2000a, p. 270). ${ }^{8}$ Sin embargo, ya desde las primeras lecciones que sostuvo en Friburgo entre 1919-1923, se pone de manifiesto la necesidad de revisar los conceptos de la fenomenología en tanto que disciplina descriptiva. Así, por ejemplo, en su primera lección sobre La idea de filosofía y el problema de la concepción del mundo (1919) advierte que «la objeción más elemental, pero cargada de suficiente peligrosidad [en contra de la investigación fenomenológica], tiene que ver con el lenguaje». Ello se debe a que «el ver fenomenológico se identifica inmediatamente con la descripción», lo cual presupone, por un lado, «la posibilidad de la formulación lingüística de lo visto» $\mathrm{y}$, por otro, «el carácter objetivante de cada lengua» (Heidegger, 2005, p. 136-137).

Esto quiere decir que en la descripción fenomenológica se pasan por alto dos presupuestos: el primero tiene que ver con la creencia en la absoluta verbalización de los fenómenos; el segundo, con la capacidad objetivante de su medio lingüístico. Con respecto al primer punto es importante aclarar que

8 Por otro lado, también es cierto que la comprensión heideggeriana del lenguaje se mueve en un extremo regionalismo, al concederle preeminencia al idioma alemán por encima de los idiomas asiáticos y latinos, a los cuales rechaza, o bien por su radical otredad (Japonés) o bien por su tendencia a la superficie y la apariencia (latín). $C f$. Volpi (2003). 
Heidegger no niega el carácter descriptivo de la fenomenología; lo que pone en tela de juicio es el hecho de que toda descripción sea tomada siempre $y$ necesariamente en términos de teorización, lo cual excluye cualquier otro tipo de intuición fundante de tipo no-teorético (2005, p. 137). En relación con la función clasificatoria y objetivante de los conceptos, afirma que ésta proviene del uso que se les da al interior de las ciencias, las cuales parten a su vez de una compresión limitada de la objetualidad, del método y del acceso.

Asumir sin cuestionar dichas funciones conceptuales en el ámbito fenomenológico implicaría, pues, articular sus objetos de modo semejante a la articulación categorial de la naturaleza. Pero los objetos de la fenomenología no son naturaleza objetiva, sino ante todo significatividad mundana, y por lo tanto, no es necesario que su expresión verbal «se piense en términos teoréticos u objetivos, sino que [sea] originariamente vivida y experimentada en un sentido mundano o en un sentido premundano» (Heidegger, 2005, p. 141). ${ }^{9}$

Con el propósito de romper con la actitud teorética también a nivel lingüístico, Heidegger introducirá en su Introducción a la fenomenología de la religión (1920/21) los así llamados indicadores o anuncios formales, es decir, conceptos no clasificatorios, y más bien aclaratorios, que sirven de guía para la explicación fenomenológica, «pero que no introdu[cen] ninguna opinión prejuzgadora en el problema»(Heidegger, 2006c, p. 81). Esto es posible porque dichos conceptos no comunican, ni mientan, ni dicen contenidos, pues no cuentan con tales; tan solo se limitan a dar una indicación, un indicio con respecto al objeto, a una determinada situación de comprensión y a quien comprende a partir de dicha situación ( $c f$. Heidegger, 2007, p. 355).

Ahora bien, dado que los signos que sirven de indicadores son signos vacíos, puesto que están ahí en lugar de lo que en sí mismo tampoco se presenta, poseen cierto carácter negativo (Heidegger, 2006c, p. 88; Inkpin, 2010, p. 25). Sin embargo, esto no quiere decir que su instauración sea arbitraria, que carezca de estructura o de dirección porque su formalidad, o mejor dicho, su forma funciona como una especie de marco, al interior del cual, ocurre el encuentro entre la indicación y aquello que ésta, por así decirlo, representa (Heidegger, 1985, p. 33). Evidentemente lo formal no se entiende aquí en contraposición a lo material, y tampoco apunta al nivel eidético del conocimiento. En lo que Heidegger denomina «un sentido existencial de lo formal», la forma de toda indicación tiene un carácter positivo y complementario en tanto que constituye el sentido referencial del fenómeno, esto es, una especie de contenido vacio

9 A la base de esta tesis, subyace otra de mayor calibre, a saber «la tesis de que la filosofía no es una ciencia teórica» (Heidegger, 2006c, p. 87). 
que permite trazar el camino para «el cumplimiento originario de lo mostrado indicativamente» $(1985$, p. 32).

Desde una perspectiva genética podríamos decir que la formación de los indicadores consta de un momento pasivo, el cual consiste en ganar la experiencia previa del objeto en su cómo fenomenológico. Este cómo, que se presenta como acceso originario al objeto, impregna -por así decirlo- al signo indicativo y le da forma, es decir, le otorga estructura y articulación. Complementario al momento pasivo de la experiencia, se da un momento activo que proviene del indicador ya formado o in-formado, y que radica en su capacidad para evocar la experiencia con vistas a su cumplimiento en un contexto de sentido dado. Dicho de otra forma: «el indicador formal gana en cada caso el espacio para su realización en y como tensión entre el camino de... y en dirección a...» (Coriando, 1988, p. 31).

Ahora bien, debido a que los indicadores formales muestran el camino hacia la experiencia comprensora, pero al mismo tiempo se originan a partir de ella, es decir, puesto que han sido formados desde el cómo fenomenológico del objeto al que apuntan originariamente; su empleo lleva implícita cierta circularidad hermenéutica (Heidegger, 1985, p. 20). Sin embargo, esta circularidad no puede ser eliminada o superada porque no se trata de un error lógico, sino que tiene lugar en el seno mismo de la interacción entre el carácter negativo de la indicación y el carácter positivo de la forma, esto es, entre la ausencia de contenido debido a su ocultamiento operativo, y el horizonte de significatividad desde el cual lo operativo se muestra como ya comprendido en su para-qué.

La única manera de salir del círculo es comprender el sesgo indicadorformal de los conceptos filosóficos como un empleo de signos, cuyo cumplimiento se lleva a cabo como una forma de praxis, debido a que su objeto no está dado completamente, sino que tiene que ser ganado en su concreción fáctica mediante la ejecución de la indicación en relación con un contexto de sentido dado (Heidegger, 2006c, p. 88; cf. Rubio, 2011, p. 87). En esto reside precisamente lo peculiar de los conceptos filosóficos ideados por Heidegger: en el hacerse comprensibles «según el cómo de la experiencia filosófica y según el cómo, en el cual la experiencia filosófica se autoexplica» (Heidegger, 1985, p. 20).

\section{CONCLUSIÓN}

En resumidas cuentas podemos decir que el tránsito del qué al cómo que caracteriza la propuesta fenomenológica desarrollada por Heidegger, consiste en una apropiación hermenéutica de la «diferencia significativa», que, de acuerdo con Waldenfels (1992), constituye en el corazón mismo de la fenomenología. Esta diferencia surge como respuesta a la pregunta por el cómo 
(wie) del darse del fenómeno y consiste en un «como» o un «en tanto que» (als). Experimentar algo en tanto que algo quiere decir que un contenido puro que se encuentre más allá de las estructuras de la conciencia o del horizonte de sentido sin posibilidad de ser determinado, sería un absurdo. En la medida en que estamos vueltos hacia las cosas de manera intencional $u$ ocupacional, éstas aparecen siempre dentro de los límites de la manifestación, es decir, siempre aparecen dotadas de cierto sentido, de cierta forma, estructura, significación o regulación (Waldenfels, 2015, p. 19).

A partir de ello, la tarea de la fenomenología puede determinarse grosso modo como una reflexión acerca de lo que se da y del modo en que se da, «pero sólo dentro de los límites de la manifestación», tal como reza el principio de todos los principios (Husserl, 1962, p. 58). Sin embargo, dicho principio será desarrollado en dos direcciones distintas: en el marco de la fenomenología trascendental de Husserl, la búsqueda del «en tanto que» será dirigida hacia la estructura del acto del significar, mientras que desde el punto de vista de la fenomenología hermenéutica de Heidegger, será retrotraída al significar fáctico del mundo.

Ahora bien, al interior del mundo entendido como horizonte de sentido, la estructura fenomenológica de algo en tanto algo se lleva a cabo como una relación de tipo $A$ como $B$ (por ejemplo: la mesa como mesa). Que $A$ se muestre como $B$ remite en primera instancia al paradigma apofántico del enunciado predicativo que se expresa con la fórmula $A$ es $B$ (la mesa es cuadrada); sin embargo, el útil que se muestra en su utilidad únicamente en el trato circunspecto, no puede volverse sujeto temático de una oración apofántica sin perder su carácter operativo. Por ello es necesario que la pregunta por el cómo de su manifestabilidad sea respondida desde una instancia conceptual acorde con la dimensión de la mirada circunspecta, en la cual la mesa es experimentada directamente como mesa, sin pasar por filtros categoriales o actos psíquicos de ningún tipo. Así, frente al como apofántico propio del enunciado verdadero, Heidegger postula un como hermenéutico previo o antepredicativo, en el cual, la mesa es lo que es, porque significa lo que significa, es decir, porque emerge desde una red significativa previamente dada y, precisamente por ello, nos resulta comprensoramente familiar.

A nivel lingüístico, el como apofántico predicativo y el como hermenéutico antepredicativo se expresan con definiciones y conceptos teoréticos y noteoréticos respectivamente. Sin embargo, para que los primeros puedan tener lugar, es necesario contar con la estructura hermenéutica del como, es decir, con la experiencia del horizonte abierto de la significatividad. (Esto quiere decir que puedo decir algo sobre la mesa solo después de haberla experimentado como tal). A partir de este giro interpretativo, el como apofántico se presenta como un decir derivado o secundario con respecto a la experiencia del como 
hermenéutico, la cual, a pesar de ser antepredicativa, no es a-lingüística. La necesidad de traer al lenguaje dicha experiencia, dará paso al desarrollo de los indicadores formales, esto es, a conceptos vacíos que señalan el camino de la experiencia fundamental correspondiente, pero sin adelantar nada acerca del contenido mismo, pues su cumplimiento consiste en la experiencia del sentido en su statu nascendi.

Así planteado, el problema de los indicadores formales parecería una repetición exuberante del planteamiento husserliano acerca del signo; sin embargo, lo interesante de la propuesta heideggeriana estriba en que, al pensar los conceptos filosóficos como directrices que apuntan a lo que permanece oculto de manera esencial, pasa por encima del modelo representacional del conocimiento que le otorga al lenguaje un papel designativo. Esto quiere decir que los conceptos de la filosofía - a diferencia de los conceptos científicos, no designan nada conocido ni traen al lenguaje nuevos descubrimientos, porque no cuentan con un referente objetual: no remiten «a algo establecido y generalmente conocido, sino que más bien intentan expresar el quebrantamiento de la solidez de lo 'bien conocido’» (Fink 1968, p. 194).

\section{REFERENCIAS BIBLIOGRÁFICAS}

BASSO, L. 2019: «La raíz óntica del sentido», Ódos Revista de filosofía VII (9), pp. 56-76.

BAUR, P. 2010: «Vom Was zum Wie. Heideggers kritik an Husserl als Neukonstitution eines dynamischen Phänomenologiebegriffs», en F. Rese (ed.), Heidegger und Husserl im Vergleich. Frankfurt: Vittorio Klostermann.

CORIANDO, P. L. 1988: «Die formale Anzeige und das Ereignis: vorbereitende Überlegungen zum Eigencharakter seinsgeschichtlicher Begrifflichkeit mit einem Ausblick auf den Unterschied von Denken und Dichten», Heidegger Studies, 14, pp. 27-43.

CIMINO, A. 2013: Phänomenologie und Vollzug. Heideggers performative Philosophie des faktischen Lebens, en F. Rese (ed.), Heidegger und Husserl im Vergleich. Frankfurt: Vittorio Klostermann.

DAHLSTROM, D. Jun.1994: «Heidegger's method: philosophical concepts as formal indications», en The Review of Metaphysics, 47 (4), pp. 775-795.

FINK, E. 1968: «Los conceptos operativos en la fenomenología de Husserl», en Husserl. Tercer Coloquio Filosófico de Royaumont. Buenos Aires: Paidós, pp.192-199.

. 1976: «Operative Begriffe in Husserls Phänomenologie», en F.-A. Schwarz (ed.), Nähe und Distanz: Phänomenologische Vorträge und Aufsätze. Freiburg/ München: Alber, pp.180-204.

GADAMER, H.-G. 2000: «La fuerza expresiva del lenguaje», en Elogio de la teoría. Barcelona: Ediciones Península, pp. 133-144.

. 2004: «La historia del concepto como filosofía», en Verdad y método II. Salamanca: Ediciones Sígueme, pp.81-94. 
GRONDIN, V. 2004: «Le langage de la phénoménologie: analogie ou citation?», en J. Benoist y S. Laugier (eds.), Husserl Et Wittgenstein: De la Description de l'Experience a la Phenomenologie Linguistique. Hildesheim: Olms.

HEIDEGGER, M. 1985: Phänomenologische Interpretationen zu Aristoteles. Einführung in die phänomenologische Forschung (WS 1921-1922) (GA 61). Frankfurt del Meno: Vittorio Klostermann.

.1993: Phänomenologie der Anschauung und des Ausdrucks (GA 59). Frankfurt del Meno: Vittorio Klostermann.

2000a: Hitos. Madrid: Alianza.

2000b: Ontología. Hermenéutica de la facticidad. Madrid: Alianza

. 2002: Interpretaciones fenomenológicas sobre Aristóteles. Madrid: Trotta

. 2003: Ser y tiempo, trad. J. E. Rivera. Madrid: Trotta.

2005: La idea de filosofía y el problema de la concepción del mundo. Barcelona: Herder.

. 2006a: Introducción a la investigación fenomenológica. Madrid: Síntesis

. 2006b: Prolegómeno sobre la historia del concepto de tiempo, Madrid: Alianza

-. 2006c: Introducción a la fenomenología de la religión. Cuidad de México: FCE

.2007: Los conceptos fundamentales de la Metafísica: mundo-soledad-finitud. Madrid: Alianza

HUSSERL, E. 1962: Ideas I. México: FCE

. 1980: Experiencia y juicio. México: UNAM.

1999a: Investigaciones lógicas I. Madrid: Alianza.

1999b: Investigaciones lógicas II. Madrid: Alianza.

IMDAHL, G. 1994: «Formale Anzeige bei Heidegger», Archiv für Begriffsgeschichte, 37, pp. 306-332

INKPIN, A. 2010: «Formale Anzeige und das Voraussetzungsproblem», en F. Rese (ed.), Heidegger und Husserl im Vergleich. Frankfurt: Vittorio Klostermann, pp. 13-33.

IONEL, L. 2017: «Sinn und Verbergung. Heideggers Theorie über die Konstitution von Bedeutsamkeit», en G. Thonhauser (ed.), Perspektiven mit Heidegger. Freiburg/München: Alber.

LUFT, S. 2002: Phänomenologie der Phänomenologie', Systematik und Methode der Phänomenologe in Auseinadersetzung zwischen Husserl und Fink. Dordrecht: Kluwer Academic Publishers.

MARTÍNEZ, P. 2008: «Hablar en silencio, decir lo indecible. Una aproximación a la cuestión de los límites del lenguaje en la obra temprana de Martin Heidegger», Diánoia, 53 (61), pp. 111-147.

ORTH, E. W. 1982: "Zur Phänomenologie des philosophischen Textes», Phänomenologische Forschungen, 12, pp. 7-20.

RODRÍGUEZ, R. 2015: Fenómeno e interpretación. Ensayos de fenomenología hermenéutica. Madrid: Tecnos.

RUBIO, R. 2011: «La doctrina de la indicación formal a la luz de la crisis del programa de 'Ser y Tiempo'», Naturaleza Humana, 13 (1), pp. 84-101.

SAN MARTIN, J. 1990: "La sexta investigación cartesiana de Eugen Fink», Revista de filosofia, Universidad Complutense, 4, pp. 247-264. 
SCHÜTZ, A. 1971: «Typus und Eidos in Husserls Spätphilosophie», en I. Schütz (ed.) Gesammelte Aufsätze III. Dordrecht: Springer, pp. 127-15.

SHOCKEY, M. 2010: «What's Formal about Formal Indication? Heidegger's Method in Sein und Zeit», Inquiry, An Interdisciplinary Journal of Philosophy, 53 (6), pp. 525-539.

VETTER, H. 2006: «Zur Begrifflichkeit der Phänomenologie am Beispiel von Husserl und Heidegger», Archiv für Begriffsgeschichte, 48, Hamburg: Felix Meiner Verlag, pp. 203-225.

VOLPI, F. 2003: "Heidegger, el problema de la intraducibilidad y la "romanitas" filosófica», en P. Brickle (coord.) La filosofía como pasión: homenaje a Jorge Rivera en su 75 cumpleaños. Madrid: Trotta, pp. 27-40.

VONGEHR, T. 2005: «Stil als Zeichen. Funktionen - Brüche - Inszenierungen», en Beiträge des 11. Internationalen Kongresses der Deutschen Gesellschaft für Semiotik (DGS), 24. Frankfurt (Oder): Schriftenreihe der EuropaUniversität Viadrina.

WALDENFELS, B. 2015: Exploraciones fenomenológicas acerca de lo extraño. Barcelona/Morelia: Anthropos/UMSNH.

. 1992: Einführung in die Phänomenologie. München: Fink.

WALTON, R. 2009: «El aparecer y lo latente», en Acta fenomenológica latinoamericana. Volumen III. Lima: Pontificia Universidad Católica del Perú-Morelia: Universidad Michoacana de San Nicolás de Hidalgo, pp. 105-120.

XOLOCOTZI, Á. 2004: Fenomenología de la vida fáctica. Heidegger y su camino a Ser y tiempo. México: Plaza y Valdés.

XOLOCOTZI, Á. (2018). Heidegger, lenguaje y escritura. México: Fontamara.

ZIRIÓN, A. y Á. XOLOCOTZI 2018: ¡A las cosas mismas! Dos ideas sobre la fenomenología. México: Benemérita Universidad Autónoma de Puebla/ Universidad Michoacana de San Nicolás de Hidalgo/Miguel Ángel Porrúa.

Ángel Xolocotzi Yáñez es profesor de Ontología en la Licenciatura en Filosofía y Seminario de problemas de Fenomenología, hermenéutica y filosofía práctica en el Posgrado de la Facultad de Filosofía y Letras, de la Benemérita Universidad Autónoma de Puebla.

Líneas de Investigación:

Fenomenología de la afectividad, Crónica de la vida y obra de Martín Heidegger, los orígenes de la fenomenología, fenomenología hermenéutica del lenguaje y la técnica.

Publicaciones recientes:

(2019): «Filosofía crítica, metafísica y fundamentación de la ciencia. Lecturas heideggerianas del neokantismo de Baden», en A. Jiménez Rodríguez (ed.). Heidegger y la historia de la filosofía: límite y posibilidad de una interpretación fenomenológica de la tradición. Granada: Editorial Comares, pp. 249-259. 
XOLOCOTZI YÁÑEZ, Á. \& ZIRIÓN QUIJANO, A. 2018: ¡A las cosas mismas! Dos ideas sobre la fenomenología. México: BUAP/Universidad Michoacana de San Nicolás de Hidalgo/Miguel Ángel Porrúa.

Correo electrónico: angel.xolocotzi@gmail.com 